\title{
Anleitung zum Lesen griechischer Wörter
}

1. Der Buchstabe 1 ist stets vokalisch zu sprechen, also nicht Jota, sondern I-ota;

2. Der Vokal $\cup$ klingt stets wie ü.

3. $\gamma$ vor $\gamma, \kappa, \xi$ und $\chi$ wird nicht wie g, sondern wie $\mathbf{n}$ ausgesprochen.

4. $\zeta$ wird stimmhaft (weich) wie ds, nicht wie ts gesprochen.

5. $\delta$ ist wie $\mathrm{t}$ zu sprechen.

6. Diphthonge oder Doppelvokale sind Zusammensetzungen der weicheren Vokale $\mathbf{~ u n d ~} v$ (hier wie $\mathbf{u}$ zu werten) mit den härteren Vokalen $\alpha$, $\varepsilon$ und $\eta$, $\circ$ und $\omega$.

Es gibt folgende Diphthonge (in Klammern die Aussprache):

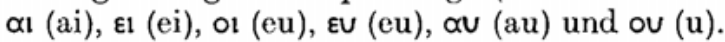

7. $\sigma$ ist stets scharf (stimmlos) zu sprechen.

Vor einem folgenden Konsonanten wird $\sigma$ stets getrennt gesprochen.

8. Es gibt 2 Hauchzeichen (spiritus). Jedes mit einem Vokal beginnende Wort hat eines dieser Hauchzeichen. Man unterscheidet einen sog. spiritus asper (') = rauher Hauch und einen spiritus lenis (') = linder Hauch. Der spiritus asper wird wie h, der spiritus lenis wird nicht gesprochen.

Bei kleiner Schrift steht der spiritus über, bei großer vor dem Buchstaben oben links. Bei Diphthongen steht der spiritus auf dem 2. Vokal.

9. Beginnt ein Wort mit $\rho$, so erhält dieses auch einen spiritus asper. Dieser wird nicht ausgesprochen.

10. Stets lang ausgesprochen werden $\eta$ und $\omega$ sowie die Diphthonge. Stets kurz auszusprechen sind: $\varepsilon$, $\circ$.

Lang oder kurz sind: $\alpha, \mathbf{1}, v$.

11. Die in einem Wort zu betonende Silbe wird durch einen Akzent gekennzeichnet. Es gibt zwei Akzente, den a cutus ('), der einen scharfen, gehobenen Ton, und den circumfléxus ( $\left.{ }^{\sim}\right)$, der einen gedehnten, breiten Ton kennzeichnet.

Eine besondere Art des acútus ist der gravis ('); er kennzeichnet einen gesenkten, gedämpften Ton. Auf der letzten Silbe eines Wortes wird der acútus durch einen gravis ersetzt, wenn noch ein Wort im Satz folgt. 